\title{
CERTAIN CONVEX HARMONIC FUNCTIONS
}

\section{YONG CHAN KIM, JAY M. JAHANGIRI, and JAE HO CHOI}

Received 21 December 2000 and in revised form 2 May 2001

We define and investigate a family of complex-valued harmonic convex univalent functions related to uniformly convex analytic functions. We obtain coefficient bounds, extreme points, distortion theorems, convolution and convex combinations for this family.

2000 Mathematics Subject Classification: 30C45, 30C50.

1. Introduction. A continuous complex-valued function $f=u+i v$ defined in a simply connected complex domain $\mathscr{D} \subset \mathbb{C}$ is said to be harmonic in $\mathscr{D}$ if both $u$ and $v$ are real harmonic in $\mathscr{D}$. Consider the functions $U$ and $V$ analytic in $\mathscr{D}$ so that $u=\mathfrak{R} U$ and $v=\mathfrak{I} V$. Then the harmonic function $f$ can be expressed by

$$
f(z)=h(z)+\overline{g(z)}, \quad z \in \mathscr{D},
$$

where $h=(U+V) / 2$ and $g=(U-V) / 2$. We call $h$ the analytic part and $g$ the coanalytic part of $f$. If the co-analytic part of $f$ is identically zero then $f$ reduces to the analytic case.

The mapping $z \mapsto f(z)$ is sense-preserving and locally one-to-one in $\mathscr{D}$ if and only if the Jacobian of $f$ is positive (see [1]), that is, if and only if

$$
J_{f}(z)=\left|h^{\prime}(z)\right|^{2}-\left|g^{\prime}(z)\right|^{2}>0, \quad z \in \mathscr{D} .
$$

Let $\mathscr{H}$ denote the family of functions $f=h+\bar{g}$ which are harmonic, sense-preserving, and univalent in the open unit disk $\Delta=\{z:|z|<1\}$ with $h(0)=f(0)=f_{z}(0)-1=0$. Thus, we may write

$$
h(z)=z+\sum_{n=2}^{\infty} a_{n} z^{n}, \quad g(z)=\sum_{n=1}^{\infty} b_{n} z^{n}, \quad\left|b_{1}\right|<1 .
$$

Also let $\overline{\mathscr{H}}$ denote the subclass of $\mathscr{H}$ consisting of functions $f=h+\bar{g}$ so that the functions $h$ and $g$ take the form

$$
h(z)=z-\sum_{n=2}^{\infty}\left|a_{n}\right| z^{n}, \quad g(z)=-\sum_{n=1}^{\infty}\left|b_{n}\right| z^{n}, \quad\left|b_{1}\right|<1 .
$$

Recently, Kanas and Wisniowska [5] (see also Kanas and Srivastava [4]), studied the class of $k$-uniformly convex analytic functions, denoted by $k$ - $U \mathscr{C} \mathscr{V}, 0 \leq k<\infty$, so that $h \in k$ - $U \mathscr{C} \mathscr{V}$ if and only if

$$
\mathfrak{R}\left\{1+(z-\zeta) \frac{h^{\prime \prime}(z)}{h^{\prime}(z)}\right\} \geq 0, \quad|\zeta| \leq k, z \in \Delta .
$$


For real $\phi$ we may let $\zeta=-k z e^{i \phi}$. Then condition (1.5) can be written as

$$
\mathfrak{R}\left\{1+\left(1+k e^{i \phi}\right) \frac{z h^{\prime \prime}(z)}{h^{\prime}(z)}\right\} \geq 0
$$

Now considering the harmonic functions $f=h+\bar{g}$ of the form (1.3) we define the family $\mathscr{H} \mathscr{C} \mathscr{V}(k, \alpha), 0 \leq \alpha<1$, so that $f=h+\bar{g} \in \mathcal{H} \mathscr{C} \mathscr{V}(k, \alpha)$ if and only if

$$
\mathfrak{R}\left\{1+\left(1+k e^{i \phi}\right) \frac{z^{2} h^{\prime \prime}(z)+\overline{2 z g^{\prime}(z)+z^{2} g^{\prime \prime}(z)}}{z h^{\prime}(z)-\overline{z g^{\prime}(z)}}\right\} \geq \alpha, \quad 0 \leq \alpha<1 .
$$

Finally, we let $\overline{\mathscr{H}} \mathscr{C} \mathscr{V}(k, \alpha) \equiv \mathscr{H} \mathscr{C} \mathscr{V}(k, \alpha) \cap \overline{\mathcal{H}}$.

Notice that if $g \equiv 0$ and $\alpha=0$ then the family $\mathscr{H} \mathscr{C} \mathcal{V}(k, \alpha)$ defined by (1.7) reduces to the class $k$ - $U \mathscr{C}^{\mathscr{V}} \mathcal{V}$ of $k$-uniformly convex analytic functions defined by (1.5). If we, further, let $k=1 \mathrm{in}(1.5)$, we obtain the class of uniformly convex analytic functions defined by Goodman [2]. A geometric characterization of the general family $\mathscr{H} \mathscr{C} \mathcal{V}(k, \alpha)$ is an open question.

In Section 2, we introduce sufficient coefficient bounds for functions to be in $\mathscr{H} \mathscr{C V}(k, \alpha)$ and show that these bounds are also necessary for functions in $\overline{\mathscr{H}} \mathscr{C} \mathscr{V}(k, \alpha)$. In Section 3, the inclusion relation between the classes $k$ - $U \mathscr{C}^{\mathscr{V}}$ and $\mathscr{H} \mathscr{C} V(k, \alpha)$ is examined. Extreme points and distortion bounds for $\mathcal{H}_{\mathfrak{C V}} \mathcal{V}(k, \alpha)$ are given in Section 4. Finally, in Section 5, we show that the class $\overline{\mathscr{H}} \mathscr{C} \mathscr{V}(k, \alpha)$ is closed under convolution and convex combinations.

Here we state a result due to Jahangiri [3], which we will use throughout this paper.

THEOREM 1.1. Let $f=h+\bar{g}$ with $h$ and $g$ of the form (1.3). If

$$
\sum_{n=2}^{\infty} \frac{n(n-\alpha)}{1-\alpha}\left|a_{n}\right|+\sum_{n=1}^{\infty} \frac{n(n+\alpha)}{1-\alpha}\left|b_{n}\right| \leq 1, \quad 0 \leq \alpha<1,
$$

then $f$ is harmonic, sense-preserving, univalent in $\Delta$, and $f$ is convex harmonic of order $\alpha$ denoted by $\mathscr{H} \mathscr{K}(\alpha)$. Condition (1.8) is also necessary if $f \in \overline{\mathscr{H}} \mathscr{K}(\alpha) \equiv \mathscr{H} \mathscr{K}(\alpha) \cap \overline{\mathscr{H}}$.

2. Coefficient bounds. First we state and prove a sufficient coefficient bound for the class $\mathscr{H}_{\mathfrak{C}} \mathcal{V}(k, \alpha)$.

THEOREM 2.1. Let $f=h+\bar{g}$ be of the form (1.3). If $0 \leq k<\infty, 0 \leq \alpha<1$, and

$$
\sum_{n=2}^{\infty} \frac{n(n+n k-k-\alpha)}{1-\alpha}\left|a_{n}\right|+\sum_{n=1}^{\infty} \frac{n(n+n k+k+\alpha)}{1-\alpha}\left|b_{n}\right| \leq 1,
$$

then $f$ is harmonic, sense-preserving, univalent in $\Delta$, and $f \in \mathscr{H}_{6} \mathcal{V}(k, \alpha)$.

Proof. Since $n-\alpha \leq n+n k-k-\alpha$ and $n+\alpha \leq n+n k+k+\alpha$ for $0 \leq k<\infty$, it follows from Theorem 1.1 that $f \in \mathscr{H} \mathscr{K}(\alpha)$ and hence $f$ is sense-preserving and convex univalent in $\Delta$. Now, we only need to show that if (2.1) holds then

$\mathfrak{R}\left\{\frac{z h^{\prime}(z)+\left(1+k e^{i \phi}\right) z^{2} h^{\prime \prime}(z)+\left(1+2 k e^{i \phi}\right) \overline{z g^{\prime}(z)}+\left(1+k e^{i \phi}\right) \overline{z^{2} g^{\prime \prime}(z)}}{z h^{\prime}(z)-\overline{z g^{\prime}(z)}}\right\}=\mathfrak{R} \frac{A(z)}{B(z)} \geq \alpha$. 
Using the fact that $\Re(w) \geq \alpha$ if and only if $|1-\alpha+w| \geq|1+\alpha-w|$ it suffices to show that

$$
|A(z)+(1-\alpha) B(z)|-|A(z)-(1+\alpha) B(z)| \geq 0,
$$

where $A(z)=z h^{\prime}(z)+\left(1+k e^{i \phi}\right) z^{2} h^{\prime \prime}(z)+\left(1+2 k e^{i \phi}\right) \overline{z g^{\prime}(z)}+\left(1+k e^{i \phi}\right) \overline{z^{2} g^{\prime \prime}(z)}$ and $B(z)=z h^{\prime}(z)-\overline{z g^{\prime}(z)}$. Substituting for $A(z)$ and $B(z)$ in (2.3), we obtain

$$
\begin{aligned}
\mid A(z)+ & (1-\alpha) B(z)|-| A(z)-(1+\alpha) B(z) \mid \\
= & \mid(2-\alpha) z+\sum_{n=2}^{\infty} n\left[n+1-\alpha+k(n-1) e^{i \phi}\right] a_{n} z^{n} \\
& +\sum_{n=1}^{\infty} n\left[n-1+\alpha+k(n+1) e^{i \phi}\right] \bar{b}_{n} \bar{z}^{n} \mid \\
& -\mid-\alpha z+\sum_{n=2}^{\infty} n\left[n-1-\alpha+k(n-1) e^{i \phi}\right] a_{n} z^{n} \\
& +\sum_{n=1}^{\infty} n\left[n+1+\alpha+k(n+1) e^{i \phi}\right] \bar{b}_{n} \bar{z}^{n} \mid \\
\geq & (2-\alpha)|z|-\sum_{n=2}^{\infty} n[n(k+1)+1-k-\alpha]\left|a_{n}\right||z|^{n} \\
& -\sum_{n=1}^{\infty} n[n(k+1)-1+k+\alpha]\left|b_{n}\right||z|^{n} \\
& -\alpha|z|-\sum_{n=2}^{\infty} n[n(k+1)-1-k-\alpha]\left|a_{n}\right||z|^{n} \\
& -\sum_{n=1}^{\infty} n[n(k+1)+1+k+\alpha]\left|b_{n}\right||z|^{n} \\
\geq 2(1-\alpha)|z|\left\{1-\sum_{n=2}^{\infty} \frac{n[n(k+1)-k-\alpha]}{1-\alpha}\left|a_{n}\right|\right. & \left.-\sum_{n=1}^{\infty} \frac{n[n(k+1)+k+\alpha]}{1-\alpha}\left|b_{n}\right|\right\} \geq 0, \quad b y(2.1) .
\end{aligned}
$$

The harmonic functions

$$
f(z)=z+\sum_{n=2}^{\infty} \frac{1-\alpha}{n(n k+n-k-\alpha)} x_{n} z^{n}+\sum_{n=1}^{\infty} \frac{1-\alpha}{n(n k+n+k+\alpha)} \bar{y}_{n} \bar{z}^{n},
$$

where $\sum_{n=2}^{\infty}\left|x_{n}\right|+\sum_{n=1}^{\infty}\left|y_{n}\right|=1$, show that the coefficient bound given in Theorem 2.1 is sharp.

The functions of the form (2.5) are in $\mathscr{H C}^{\mathscr{V} V}(k, \alpha)$ because

$$
\sum_{n=2}^{\infty} \frac{n(n+n k-k-\alpha)}{1-\alpha}\left|a_{n}\right|+\sum_{n=1}^{\infty} \frac{n(n+n k+k+\alpha)}{1-\alpha}\left|b_{n}\right|=\sum_{n=2}^{\infty}\left|x_{n}\right|+\sum_{n=1}^{\infty}\left|y_{n}\right|=1
$$


Next we show that the bound (2.1) is also necessary for functions in $\overline{\mathscr{H}} \mathscr{C} \mathscr{V}(k, \alpha)$.

THEOREM 2.2. Let $f=h+\bar{g}$ with $h$ and $g$ of the form (1.4). Then $f \in \overline{\mathscr{H}} \mathscr{C V}(k, \alpha)$ if and only if

$$
\sum_{n=2}^{\infty} \frac{n(n+n k-k-\alpha)}{1-\alpha}\left|a_{n}\right|+\sum_{n=1}^{\infty} \frac{n(n+n k+k+\alpha)}{1-\alpha}\left|b_{n}\right| \leq 1
$$

Proof. In view of Theorem 2.1, we only need to show that $f \notin \overline{\mathscr{H}} \mathscr{C} \mathscr{V}(k, \alpha)$ if condition (2.7) does not hold. We note that a necessary and sufficient condition for $f=h+\bar{g}$ given by (1.4) to be in $\overline{\mathcal{H}} \mathscr{C} \mathscr{V}(k, \alpha)$ is that the coefficient condition (1.7) to be satisfied. Equivalently, we must have

$$
\mathfrak{R} \frac{(1-\alpha) z h^{\prime}(z)+\left(1+k e^{i \phi}\right) z^{2} h^{\prime \prime}(z)+\left(1+\alpha+2 k e^{i \phi}\right) \overline{z g^{\prime}(z)}+\left(1+k e^{i \phi}\right) \overline{z^{2} g^{\prime \prime}(z)}}{z h^{\prime}(z)-\overline{z g^{\prime}(z)}} \geq 0 .
$$

Upon choosing the values of $z$ on the positive real axis where $0 \leq z=r<1$, the above inequality reduces to

$$
\frac{1-\alpha-\left\{\sum_{n=2}^{\infty} n(n k+n-k-\alpha)\left|a_{n}\right|+\sum_{n=1}^{\infty} n(n k+n+k+\alpha)\left|b_{n}\right|\right\} r^{n-1}}{1-\sum_{n=2}^{\infty} n\left|a_{n}\right| r^{n-1}+\sum_{n=1}^{\infty} n\left|b_{n}\right| r^{n-1}} \geq 0 .
$$

If condition (2.7) does not hold then the numerator in (2.9) is negative for $r$ sufficiently close to 1 . Thus there exists $z_{0}=r_{0}$ in $(0,1)$ for which the quotient (2.9) is

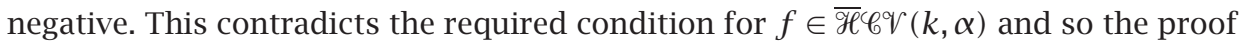
is complete.

3. Inclusion relations. As mentioned earlier in the proof of Theorem 2.1, the functions in $\overline{\mathscr{H}} \mathscr{C} V(k, \alpha)$ are convex harmonic in $\Delta$. In the following example we show that this inclusion is proper.

EXAMPLE 3.1. Consider the harmonic functions

$$
f_{n}(z)=z-\frac{1}{2} \bar{z}-\frac{1}{2 n^{2}} \bar{z}^{n}, \quad z \in \Delta, n=2,3, \ldots
$$

For $a_{n} \equiv 0$ and $b_{n}=-1 / 2 n^{2}$, we observe that

$$
\sum_{n=2}^{\infty} n^{2}\left|a_{n}\right|+\sum_{n=1}^{\infty} n^{2}\left|b_{n}\right|=\frac{1}{2}+n^{2}\left(\frac{1}{2 n^{2}}\right)=\frac{1}{2}+\frac{1}{2}=1 .
$$

Therefore, by Theorem 1.1, $f_{n} \in \overline{\mathscr{H}} \mathscr{H}(0)$.

On the other hand,

$$
\frac{2 k+1+\alpha}{1-\alpha}\left|-\frac{1}{2}\right|+\frac{n(n k+n+k+\alpha)}{1-\alpha}\left|-\frac{1}{2 n}\right|=\frac{2 k+1+\alpha}{2(1-\alpha)}+\frac{n k+n+k+\alpha}{2 n(1-\alpha)}>1 .
$$

Thus, by Theorem 2.2, $f \notin \overline{\mathscr{H}} \mathscr{C} \mathscr{V}(k, \alpha)$.

More generally, we can prove the following theorem.

THEOREM 3.2. Let $0 \leq k<\infty, 0 \leq \alpha<1$, and $0 \leq \beta<1$. If $k>\beta /(1-\beta)$ then the proper inclusion relation $\overline{\mathscr{H}} \mathscr{C} \mathscr{V}(k, \alpha) \subset \overline{\mathscr{H}} \mathscr{H}(\beta)$. 
Proof. Let $f \in \overline{\mathcal{H}} \mathscr{C} \mathfrak{V}(k, \alpha)$, then, by Theorem 2.2,

$$
\sum_{n=2}^{\infty} \frac{n(n k+n-k-\alpha)}{1-\alpha}\left|a_{n}\right|+\sum_{n=1}^{\infty} \frac{n(n k+n+k+\alpha)}{1-\alpha}\left|b_{n}\right| \leq 1
$$

Since $(n-\beta) /(1-\beta)<(n k+n-k-\alpha) /(1-\alpha)$ and $(n+\beta) /(1-\beta)<(n k+n+$ $k+\alpha) /(1-\alpha)$, by Theorem 1.1, we conclude that $f \in \overline{\mathscr{H}} \mathscr{K}(\beta)$.

To show that the inclusion is proper, consider the harmonic functions

$$
f_{n}(z)=z-\frac{1-\beta}{2(1+\beta)} \bar{z}-\frac{1-\beta}{2 n(n+\beta)} \bar{z}^{n}, \quad z \in \Delta, n=2,3, \ldots
$$

By Theorem 1.1, $f_{n} \in \overline{\mathscr{H}} \mathscr{H}(\beta)$, because

$$
\sum_{n=2}^{\infty} \frac{n(n-\beta)}{1-\beta}\left|a_{n}\right|+\sum_{n=1}^{\infty} \frac{n(n+\beta)}{1-\beta}\left|b_{n}\right|=\frac{1+\beta}{1-\beta} \frac{1-\beta}{2(1+\beta)}+\frac{n(n+\beta)}{1-\beta} \frac{1-\beta}{2 n(n+\beta)}=1 .
$$

On the contrary, by Theorem 2.2, $f_{n} \notin \overline{\mathscr{H}} \mathscr{C} \mathscr{V}(k, \alpha)$, because

$$
\begin{aligned}
\sum_{n=1}^{\infty} \frac{n(n k+n+k+\alpha)}{1-\alpha}\left|b_{n}\right| & =\frac{1+\alpha+2 k}{1-\alpha} \frac{1-\beta}{2(1+\beta)}+\frac{n(n+\alpha+(n+1) k)}{1-\alpha} \frac{1-\beta}{2 n(n+\beta)} \\
& =\frac{1-\beta}{2(1-\alpha)}\left\{\frac{1+\alpha+2 k}{1+\beta}+\frac{n+\alpha+(n+1) k}{n+\beta}\right\} \\
& >\frac{1-\beta}{2(1-\alpha)}\left\{\frac{1+\alpha+2 \beta /(1-\beta)}{1+\beta}+\frac{n+\alpha+(n+1) \beta /(1-\beta)}{n+\beta}\right\} \\
& =\frac{1}{2(1-\alpha)}\left\{2+\frac{\alpha(1-\beta)(n+1+2 \beta)}{(1+\beta)(n+\beta)}\right\} \geq 1 .
\end{aligned}
$$

4. Extreme points and distortion bounds. Using definition (1.7), and according to the arguments given in [3], we obtain the following extreme points of the closed convex hulls of $\overline{\mathscr{H}} \mathscr{C} \mathscr{V}(k, \alpha)$ denoted by $\overline{\overline{c l}} \overline{\bar{c}} \overline{\mathscr{H}} \mathscr{C} \mathscr{V}(k, \alpha)$.

THEOREM 4.1. Let $f$ be the form of (1.4). Then $f \in \overline{\mathrm{cl}} \overline{\overline{c o}} \mathscr{C O}(k, \alpha)$ if and only if $f(z)=\sum_{n=1}^{\infty}\left(X_{n} h_{n}+Y_{n} g_{n}\right)$ where $h_{1}(z)=z, h_{n}(z)=z-((1-\alpha) / n(n+n k-$ $k-\alpha)) z^{n}(n=2,3, \ldots), g_{n}(z)=z-((1-\alpha) / n(n+n k+k+\alpha)) \bar{z}^{n}(n=1,2,3, \ldots)$, $\sum_{n=1}^{\infty}\left(X_{n}+Y_{n}\right)=1, X_{n} \geq 0$ and $Y_{n} \geq 0$. In particular, the extreme points of $\overline{\mathscr{H}} \mathscr{C V}(k, \alpha)$ are $\left\{h_{n}\right\}$ and $\left\{g_{n}\right\}$.

Similarly, follows the distortion bounds for functions in $\overline{\mathscr{H}} \mathscr{C} \mathscr{V}(k, \alpha)$.

THEOREM 4.2. If $f \in \overline{\mathcal{H}} \mathscr{C} V(k, \alpha)$ then

$$
\begin{aligned}
& |f(z)| \leq\left(1+\left|b_{1}\right|\right) r+\frac{1}{2}\left(\frac{1-\alpha}{2+k-\alpha}-\frac{1+2 k+\alpha}{2+k-\alpha}\left|b_{1}\right|\right) r^{2}, \quad|z|=r<1, \\
& |f(z)| \geq\left(1-\left|b_{1}\right|\right) r-\frac{1}{2}\left(\frac{1-\alpha}{2+k-\alpha}-\frac{1+2 k+\alpha}{2+k-\alpha}\left|b_{1}\right|\right) r^{2}, \quad|z|=r<1 .
\end{aligned}
$$


If we let $r \rightarrow 1$ in the left-hand inequality of Theorem 4.2 and collect the like terms, we obtain the following theorem.

THEOREM 4.3. If $f \in \overline{\mathscr{H}} \mathscr{C} \mathcal{V}(k, \alpha)$ then $\{w:|w|<(3+2 k-\alpha) / 2(2+k-\alpha)-3(1-\alpha) /$ $\left.2(2+k-\alpha)\left|b_{1}\right|\right\} \subset f(\Delta)$.

5. Convolutions and convex combinations. For harmonic functions $f(z)=z-$ $\sum_{n=2}^{\infty}\left|a_{n}\right| z^{n}-\sum_{n=1}^{\infty}\left|b_{n}\right| \bar{z}^{n}$ and $F(z)=z-\sum_{n=2}^{\infty}\left|A_{n}\right| z^{n}-\sum_{n=1}^{\infty}\left|B_{n}\right| \bar{z}^{n}$, we define the convolution of $f$ and $F$ as

$$
(f * F)(z)=f(z) * F(z)=z-\sum_{n=2}^{\infty}\left|a_{n}\right|\left|A_{n}\right| z^{n}-\sum_{n=1}^{\infty}\left|b_{n}\right|\left|B_{n}\right| \bar{z}^{n} .
$$

In the following theorem we examine the convolution properties of the class $\overline{\mathscr{H}} \mathscr{C} \mathfrak{V}(k, \alpha)$.

THEOREM 5.1. For $0 \leq \alpha \leq \beta<1$, let $f \in \overline{\mathscr{H}} \mathscr{C} \mathscr{V}(k, \beta)$ and $F \in \overline{\mathscr{H}} \mathscr{C} \mathscr{V}(k, \alpha)$ then

$$
f * F \in \overline{\mathscr{H}} \mathscr{C} \mathscr{V}(k, \beta) \subset \overline{\mathscr{H}} \mathscr{C} \mathscr{V}(k, \alpha) .
$$

Proof. Express the convolution of $f$ and $F$ as that given by (5.1) and note that $\left|A_{n}\right| \leq 1$ and $\left|B_{n}\right| \leq 1$. Now the theorem follows upon the application of the required condition (2.7).

The convex combination properties of the class $\overline{\mathscr{H}} \mathscr{C} \mathscr{V}(k, \alpha)$ is given in the following theorem.

THEOREM 5.2. The class $\overline{\mathscr{H}} \mathscr{C} \mathcal{V}(k, \alpha)$ is closed under convex combinations.

Proof. For $i=1,2, \ldots$, suppose that $f_{i} \in \overline{\mathscr{H}} \mathscr{C} \mathscr{V}(k, \alpha)$ where $f_{i}$ is given by $f_{i}(z)=$ $z-\sum_{n=2}^{\infty}\left|a_{i_{n}}\right| z^{n}-\sum_{n=1}^{\infty}\left|b_{i_{n}}\right| \bar{z}^{n}$. For $\sum_{i=1}^{\infty} t_{i}=1,0 \leq t_{i} \leq 1$, the convex combinations of $f_{i}$ may be written as

$$
\sum_{i=1}^{\infty} t_{i} f_{i}(z)=z-\sum_{n=2}^{\infty}\left(\sum_{i=1}^{\infty} t_{i}\left|a_{i_{n}}\right|\right) z^{n}-\sum_{n=1}^{\infty}\left(\sum_{i=1}^{\infty} t_{i}\left|b_{i_{n}}\right|\right) \bar{z}^{n} .
$$

Now, the theorem follows by (2.7) upon noting that $\sum_{i=1}^{\infty} t_{i}=1$.

ACKNOWLEDGMENT. The research of the first author was supported by Yeungnam University, Korea.

\section{REFERENCES}

[1] J. Clunie and T. Sheil-Small, Harmonic univalent functions, Ann. Acad. Sci. Fenn. Ser. A I Math. 9 (1984), 3-25.

[2] A. W. Goodman, On uniformly convex functions, Ann. Polon. Math. 56 (1991), no. 1, 87-92.

[3] J. M. Jahangiri, Coefficient bounds and univalence criteria for harmonic functions with negative coefficients, Ann. Univ. Mariae Curie-Skłodowska Sect. A 52 (1998), no. 2, $57-66$.

[4] S. Kanas and H. M. Srivastava, Linear operators associated with $k$-uniformly convex functions, Integral Transform. Spec. Funct. 9 (2000), no. 2, 121-132. 
[5] S. Kanas and A. Wisniowska, Conic regions and k-uniform convexity, J. Comput. Appl. Math. 105 (1999), no. 1-2, 327-336.

Yong Chan Kim: Department of Mathematics Education, Yeungnam University, GYONGSAN 712-749, KOREA

E-mail address: kimyc@yu.ac.kr

Jay M. Jahangiri: Department of Mathematics, Kent State University, Burton, OH 44021-9500, USA

E-mail address: jay@geauga . kent. edu

Jae Ho Choi: Department of Mathematics Education, Yeungnam University, GYONGSAN 712-749, KOREA 


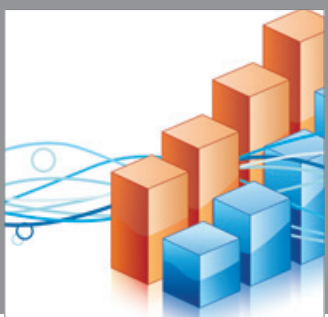

Advances in

Operations Research

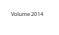

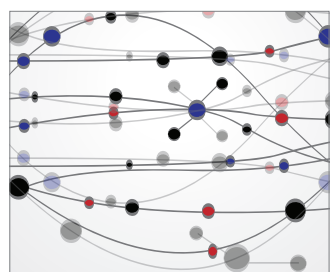

\section{The Scientific} World Journal
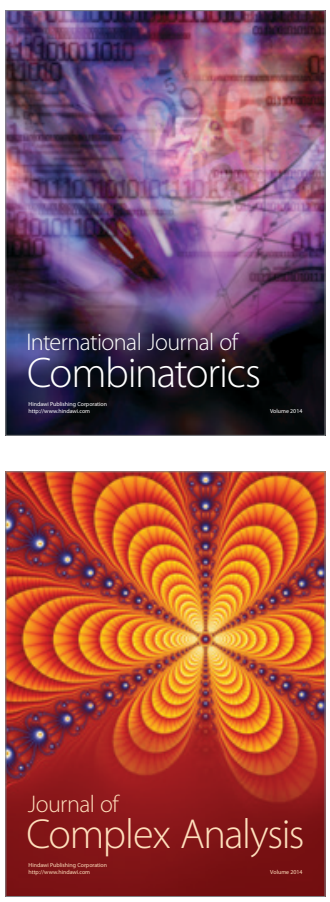

International Journal of

Mathematics and

Mathematical

Sciences
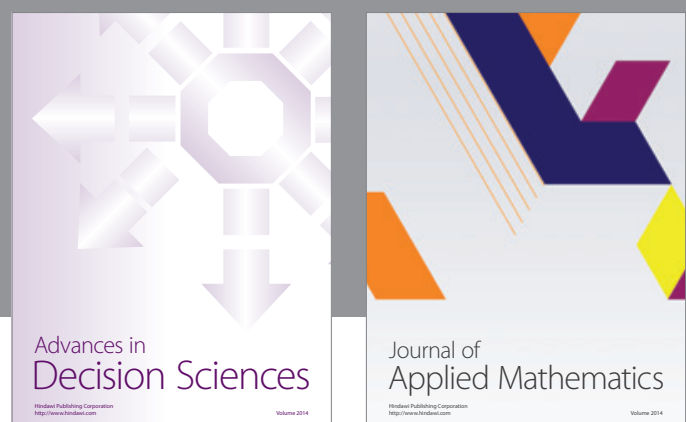

Journal of

Applied Mathematics
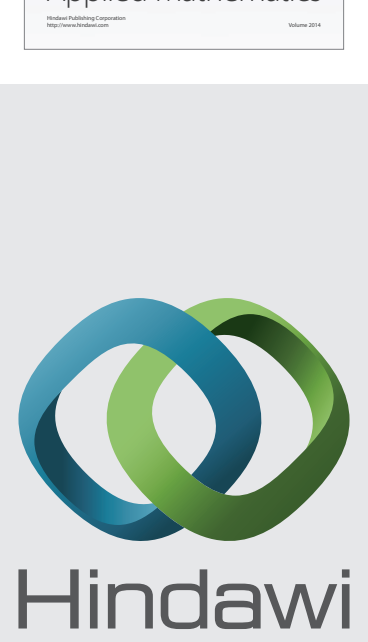

Submit your manuscripts at http://www.hindawi.com
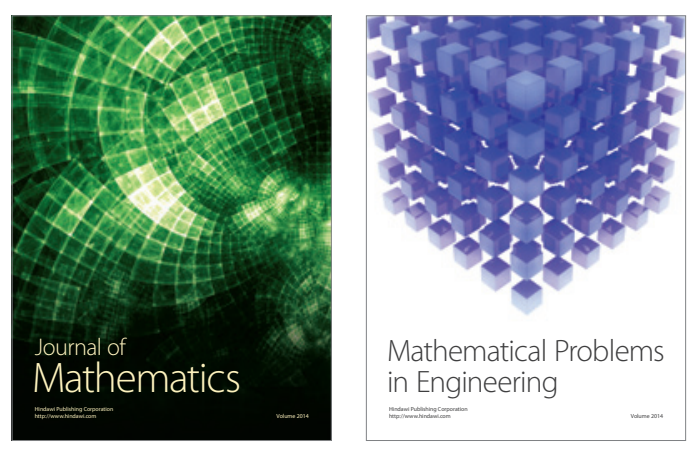

Mathematical Problems in Engineering
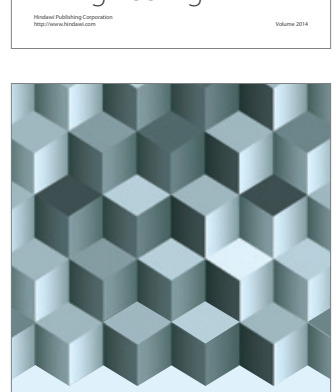

Journal of

Function Spaces
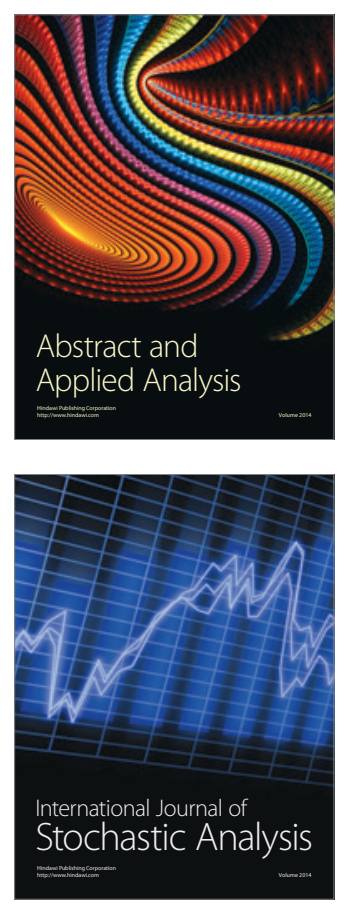

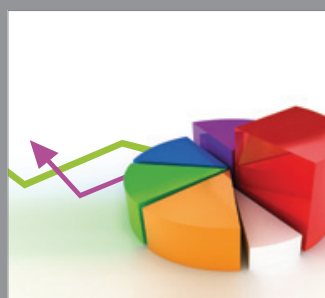

ournal of

Probability and Statistics

Promensencen
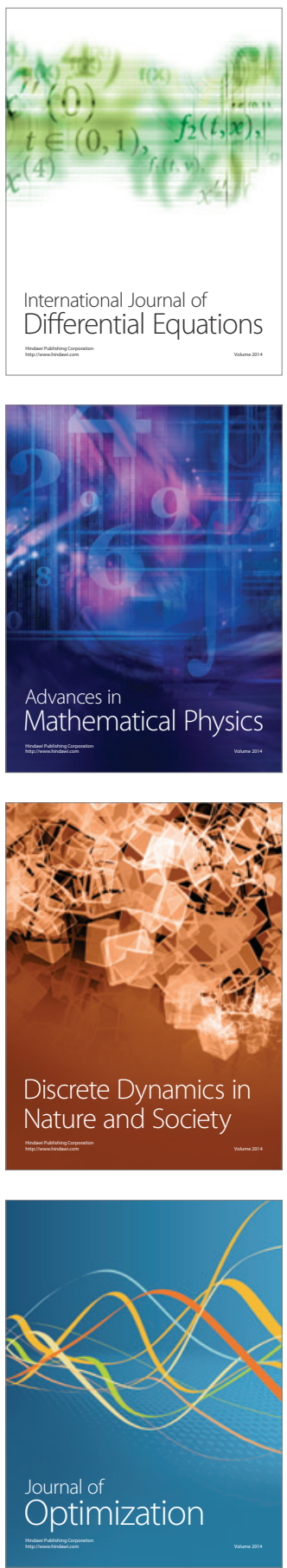